\title{
TRIC: A Thermal Resistance and Impedance Calculator for Electronic Packages ${ }^{\dagger}$
}

\author{
Lorenzo Codecasa ${ }^{1}$, Francesca De Viti ${ }^{2}$, Vincenzo d'Alessandro ${ }^{3, *}$, Donata Gualandris ${ }^{2}$, \\ Arianna Morelli ${ }^{2}$ and Claudio Maria Villa ${ }^{2}$ \\ 1 Department of Electronics, Information and Bioengineering, Politecnico di Milano, 20133 Milan, Italy; \\ lorenzo.codecasa@polimi.it \\ 2 STMicroelectronics, 20864 Agrate Brianza, Italy; francesca.deviti@st.com (F.D.V.); \\ donata.gualandris@st.com (D.G.); arianna.morelli@st.com (A.M.); claudio-maria.villa@st.com (C.M.V.) \\ 3 Department of Electrical Engineering and Information Technology, University Federico II, 80125 Naples, Italy \\ * Correspondence: vindales@unina.it \\ + This manuscript is based on the conference paper "Thermal Resistance and Impedance Calculator (TRIC)" \\ included in the Proceedings of the 25th International Workshop on Thermal Investigations of ICs and \\ Systems, Lecco, Italy, 25-27 September 2019.
}

Received: 21 February 2020; Accepted: 21 April 2020; Published: 4 May 2020

\begin{abstract}
This paper presents the Thermal Resistance and Impedance Calculator (TRIC) tool devised for the automatic extraction of thermal metrics of package families of electronic components in both static and transient conditions. TRIC relies on a solution algorithm based on a novel projection-based approach, which —unlike previous techniques-allows (i) dealing with parametric detailed thermal models (pDTMs) of package families that exhibit generic non-Manhattan variations of geometries and meshes, and (ii) transforming such pDTMs into compact thermal models that can be solved in short times. Thermal models of several package families are available, and dies with multiple active areas can be handled. It is shown that transient thermal responses of chosen packages can be obtained in a CPU (central processing unit) time much shorter than that required by a widely used software relying on the finite-volume method without sacrificing accuracy.
\end{abstract}

Keywords: electronic packages; detailed thermal model; Joint Electron Device Engineering Council (JEDEC) metrics; thermal impedance; thermal simulation

\section{Introduction}

The thermal analysis of electronic devices, circuits, and systems has always been an activity of utmost relevance in the semiconductor industry and academia. A thermally aware design can be achieved with the aid of numerical simulations, which are very challenging in terms of CPU time and memory storage, if a high level of accuracy is desired. This has stimulated many research groups to develop tools relying on suitable algorithms to accelerate the solution process (e.g., [1-3]).

For the specific case of packaged components, the preferred approach is to build boundary condition independent (BCI) compact thermal models (CTMs) to alleviate the computational burden without perceptible accuracy loss [4-7]; an interesting review of BCI CTMs for electronic parts is offered in [8].

Standardized procedures have been introduced to allow a fair comparison among packaged components in terms of thermal performances. More specifically, Joint Electron Device Engineering Council (JEDEC) metrics [9] are evaluated and included in the product datasheets. In order to satisfy the request for JEDEC thermal metrics of chosen package families, the authors developed the Thermal Resistance Advanced Calculator (TRAC) tool, the features of which were initially sketched in [10] 
and then fully described in [11]. TRAC was conceived (i) to allow a straightforward definition of a parametric detailed thermal model (pDTM) of a package with Manhattan geometry/mesh (as well as Manhattan geometry/mesh variations) and (ii) to automatically determine the thermal metrics of the package from the simulated (static) temperature field. TRAC makes use of a model-order reduction (MOR) technique (e.g., [12-23]) to transform the pDTM into a CTM, which can be solved in a short time. Various package families were covered, namely, exposed-pad (epad) low-profile (thick) and thin quad flat packages (eLQFPs and eTQFPs, respectively) as well as exposed-pad quad-flat no-leads (eQFN) packages. However, only one heat source (HS) with arbitrary size and position could be activated within the semiconductor die.

TRAC was believed to fulfill the requests of vendors in the semiconductor industry. It has the potential to allow people not necessarily endowed with expertise in the thermal field (such as system engineers, marketing people, etc.) to easily get the thermal metrics of the packages of interest. Additionally, it is suited to free thermal experts from these standard and repetitive tasks, giving them the possibility to focus on crucial thermal issues.

However, as additional package families were considered, soon emerged the need to deal with variations in geometries/meshes much more complex than those manageable by MOR methods like the one implemented in TRAC. For this reason, a new simulation tool referred to as Thermal Resistance and Impedance Calculator (TRIC) [24] has been realized, which enriches the functionalities of TRAC as follows: (i) TRIC relies on a novel advanced projection-based approach that allows deriving CTMs from the pDTMs of package families with Manhattan geometry/mesh and generic non-Manhattan geometry/mesh transformations without efficiency loss; (ii) in addition to the package families available in TRAC, also full-plastic LQFPs (pLQFPs) are included; (iii) the temperature field can also be evaluated under transient conditions; (iv) the pDTMs and the related CTMs of components with multiple HSs can be generated and simulated. It must be remarked that for both pLQFPs and multi-source packages the nature of the geometry/mesh variations is inherently non-Manhattan.

After the contribution [24] was presented, a new TRIC version was released, which also covers eQFN packages with multiple rows of pins (eQFN-mr), as well as PowerSSO packages. Consequently, such a release can handle pDTMs corresponding to a massive amount of package families, which is destined to further increase in the near future.

The aim of this paper is to extend [24] by

- providing an exhaustive picture of the TRIC features;

- reporting and discussing a larger number of results, including those obtained for the newly included package families (eQFN-mr and PowerSSO);

- adding a detailed comparison (only touched upon in [24]) in terms of accuracy and CPU time with the commercial finite-volume (FV) software FloTHERM [25];

- showing a simulated temperature map at a chosen time instant for a multi-source case of practical relevance.

The remainder of the paper is articulated as follows. In Section 2, TRIC is described and the details concerning all the thermally-modeled packages are provided. Section 3 probes into the solution algorithm. The numerical results and the main findings, as well as the comparison with FloTHERM, are shown and discussed in Section 4. Conclusions are then given in Section 5.

\section{TRIC Features}

TRIC, like the former release TRAC, is suited to automatically extract the JEDEC metrics $\vartheta_{\text {JA }}$, $\Psi_{\mathrm{JB}}, \Psi_{\mathrm{JCtop}}, \vartheta_{\mathrm{JB}}, \vartheta_{\mathrm{JCtop}}$, and $\vartheta_{\mathrm{JCbottom}}$ in four ambients [9] for each specimen in a package family. The ambients mainly differ in terms of thermal path followed by the heat generated within the HS and emerging from the die; more specifically, the ambient to evaluate $\vartheta_{\text {JCbottom }}$ requires a cold plate in intimate contact with the package backside; the plate is located over the top surface when aiming to compute $\vartheta_{\mathrm{JCtop}}$; in the ambient for determining $\vartheta_{\mathrm{JB}}$, a cold ring surrounds the package; no cooling 
systems are adopted in the ambient common to $\vartheta_{\mathrm{JA}}, \Psi_{\mathrm{JB}}$, and $\Psi_{\mathrm{JCtop}}$. To this end, a purely conductive pDTM is defined for each family of packages immersed in a specific ambient, within which the boundary conditions (BCs) are calibrated following the JEDEC environment specifications. For instance, for the evaluation of $\vartheta_{\text {JCbottom }}$, an extremely high heat transfer coefficient was applied to the bottom surface to describe the thermal path from the die to the heat sink. The geometry, assumed to be Manhattan (whereas its variation can also be non-Manhattan), is modeled by subdividing the domain into rectangular parallelepipeds (also referred to as cells or, more picturesquely, as bricks) with edges parallel to the $\mathrm{x}, \mathrm{y}$, and $\mathrm{z}$ axes, for which dimensions, material properties, and heat generation are provided. All data on geometry and properties are stored in a parameter vector $\mathbf{p}$ varying in a set P. The pDTM includes information to automatically generate a Cartesian mesh for all specimens belonging to a family of packages in the chosen ambient.

It is worth noting that the pDTMs are created by resorting to reasonable simplifications (coming from the vendor's experience) that allow a marked reduction in computational burden while negligibly affecting the simulation accuracy. More specifically, in all ambients, the board over which the package is mounted is modeled with a single finely-meshed parallelepiped with a thermal conductivity adjusted to account for the aggregate effect of metal traces and vias, the detailed representation of which would have led to a far too complex problem. In a similar fashion, the pins in the eQFN and eQFN-mr package families, as well as the leads in the pLQFP and PowerSSO families, were thermally represented by a rectangular parallelepiped, the thermal conductivity of which was determined through a weighted averaging procedure.

The thermal metrics can be automatically evaluated through a graphical user interface, in which a chosen specimen in a package family is determined by selecting the corresponding set of parameters. Package size and thickness, pad size, lead count, die size, and thickness are examples of the data that the user can input.

As mentioned in [11], a preliminary convergence analysis of the 3-D mesh discretization of the constructed pDTMs was performed for selected packages; in particular, the calculated thermal metrics were monitored by increasing the degrees of freedom (DoF) until a negligible mesh sensitivity was observed. Then the discretization leading to about $0.1 \%$ inaccuracy was chosen to avoid unnecessarily onerous too-fine meshes.

So far, the pDTMs of many package families are available. The list, along with the main geometrical features, is reported below.

- eQFPs, which are surface mount integrated circuit packages with a flat rectangular body, and leads extending from all the four sides. In particular, both the eLQFP and eTQFP variants are available, which differ in terms of body thickness (1.4 and $1 \mathrm{~mm}$, respectively). The square epad structure, which is a standard lead frame wherein the die pad is depressed down to the package bottom face, represents a valuable solution to ease the heat dissipation from die to board. The horizontal size of the body can be $7 \times 7,10 \times 10,14 \times 14,20 \times 20,24 \times 24 \mathrm{~mm}^{2}$, the total number of leads can be 32, 48, 64, 80, 100, 128, 144, 176, 216 for both variants; several sizes for the epad are available, which span from $3.5 \times 3.5$ to $9 \times 9 \mathrm{~mm}^{2}$. Various types of glue for die attach can be selected. The die thickness can amount to 100, 280, 375 (used in the simulations shown in Section 4), and $580 \mu \mathrm{m}$ (the latter value for the eLQFPs only), while any technologically-reasonable horizontal size can be chosen. It is worth noting that only parameter sets corresponding to real packages fabricated by STMicroelectronics can be selected, whereas all other combinations are obviously not possible; examples are: the $10 \times 10 \mathrm{~mm}^{2}$ eTQFP with 80 leads can be equipped only with an epad with sizes $3.5 \times 3.5,5.4 \times 5.4,6.2 \times 6.2 \mathrm{~mm}^{2}$; many sizes are instead possible for the epad in the $14 \times 14 \mathrm{~mm}^{2}$ eLQFPs with 100 leads, namely, $3.5 \times 3.5,4.5 \times 4.5,6.0 \times 6.0,7.2 \times 7.2$, $7.6 \times 7.6,8.5 \times 8.5 \mathrm{~mm}^{2} ; 32,40$, and 48 leads are available only for the $7 \times 7 \mathrm{~mm}^{2}$ eLQFP.

- pLQFPs, where, contrary to the eLQFP counterparts, the mold covers the entire package surface, so that the metal base of the lead frame is not "exposed" and thus not visible from the package bottom. With a few exceptions, all the parameter sets already reported for the eLQFPs are possible. 
- eQFN packages, which are lead-less flat molded structures built with a metal lead-frame manufactured by etching, and represent a popular cost-effective and high-performance packaging solution by virtue of the lower inductance than in leaded packages. Several horizontal sizes of the square package body are available, spanning from $2 \times 2$ to $15 \times 15 \mathrm{~mm}^{2}$, while the thickness can be equal to $0.55,0.75$, and $0.9 \mathrm{~mm}$ (the latter being adopted for the simulations in Section 4 ). The horizontal sizes of epad and die can be arbitrarily chosen in the ranges allowed by the design rules. Various types of die attach can be selected. Specimens of this family can be equipped with a single row of pins (single-row QFN) or with multiple rows of pins (eQFN-mr), this option being not available in the former TRIC version.

- PowerSSO packages, which are derived from the well-known Small Outline (SO) family and benefit from footprint and height 30\%-50\% smaller than a conventional dual in-line package. More specifically, epad PowerSSO structures are considered, which are conceived to favor the heat removal without extra cost penalty. Many packages belonging to the PowerSSO family have been included, namely (i) PowerSSO-12, PowerSSO-14, and PowerSSO-16, all sharing a $4.9 \times 3.9 \times 1.5 \mathrm{~mm}^{3}$ body and equipped with 12 leads (the lead pitch being $0.8 \mathrm{~mm}$ ), 14 leads $(0.65 \mathrm{~mm})$, and 16 leads $(0.5 \mathrm{~mm})$, respectively; (ii) PowerSSO-24, PowerSSO-28, and PowerSSO-36, all sharing a $7.5 \times 10.3 \times 2.3 \mathrm{~mm}^{3}$ body, and equipped with 24 leads (the lead pitch being $0.8 \mathrm{~mm}$ ), 28 leads $(0.65 \mathrm{~mm})$, and 36 leads $(0.5 \mathrm{~mm})$, respectively. PowerSSO packages were not covered by the first TRIC release.

Bottom side views of the above-reported packages are shown in Figure 1, while examples of DTMs are depicted in Figure 2. Thermal models of further electronic components can be created with relatively little effort.

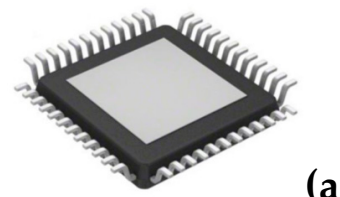

(a)

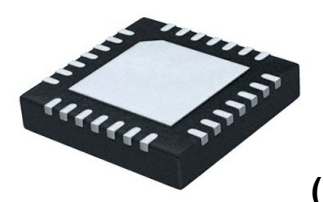

(c)

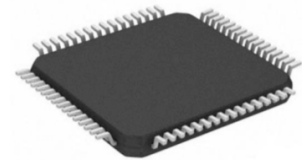

(b)

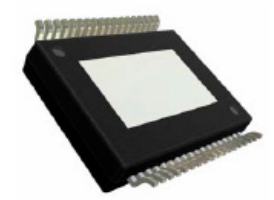

(d)

Figure 1. Bottom side views of specimens of the (a) eLQFP, (b) pLQFP, (c) single-row eQFN, and (d) PowerSSO-36 package families.
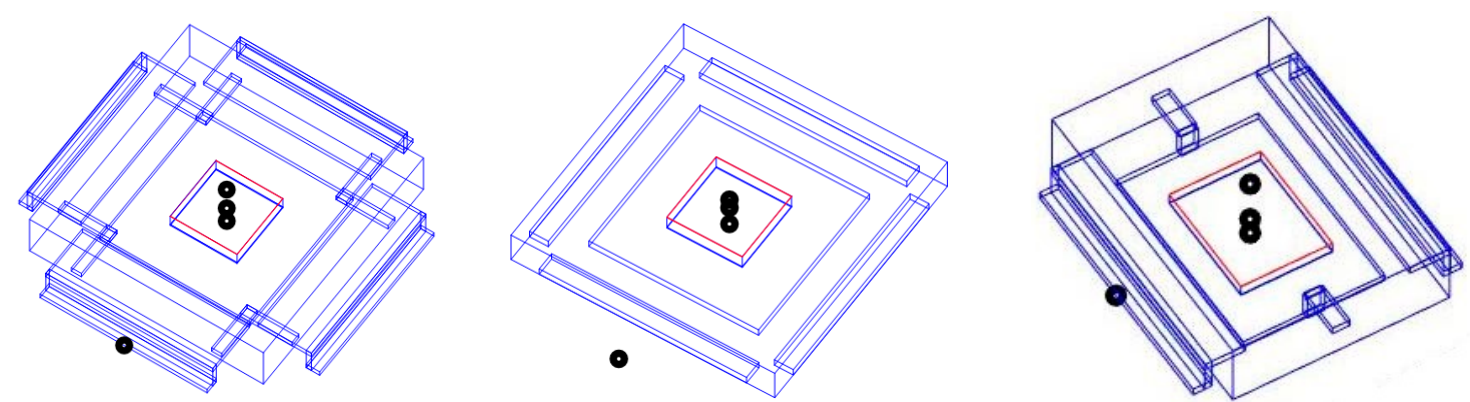

Figure 2. DTMs of the eLQFP (left), single-row eQFN (center), and PowerSSO-36 (right) families. The black circles represent the temperature probes needed to determine the thermal metrics.

Differently from TRAC, in TRIC the evaluation of the temperature field under transient conditions can be enabled for any profile of dissipated power at the HSs. In addition, TRIC allows coping with die 
layouts integrating circuitries with multiple separate active areas (i.e., HSs), for which the assumption of a uniform power distribution over the whole die surface would have been unacceptably inaccurate. As a result, TRIC can be used with many practical aims. As an example, by emulating the dissipation over the die with only one HS, the thermal impedance can be computed by determining the thermal response to a power step. Moreover, power profiles typically encountered in real applications, like the antilock braking system (ABS) in vehicles, injection, etc., can be taken into account.

\section{TRIC Solution Algorithm}

The parametric MOR techniques implemented in TRAC are only suited to deal with Manhattan variations of geometry/mesh. A Manhattan transformation is a geometry transformation that converts the $\mathrm{x}, \mathrm{y}$, and $\mathrm{z}$ coordinates into coordinates $X, Y$, and $Z$ given by

$$
\begin{aligned}
& X=f_{x}(x) \\
& Y=f_{y}(y) \\
& Z=f_{z}(z)
\end{aligned}
$$

with $f_{x}, f_{y}$, and $f_{z}$ continuous and monotonic functions. Conveniently, contrary to TRAC, TRIC exploits a two-step approach to cope with Manhattan pDTMs associated with non-Manhattan variations of geometry/mesh. This is, for example, the case (i) of pLQFPs, where the relative position of the leads changes by varying the die thickness (Figure 3), and (ii) of multi-source dies, where the relative position of the HSs can be modified (Figure 4).

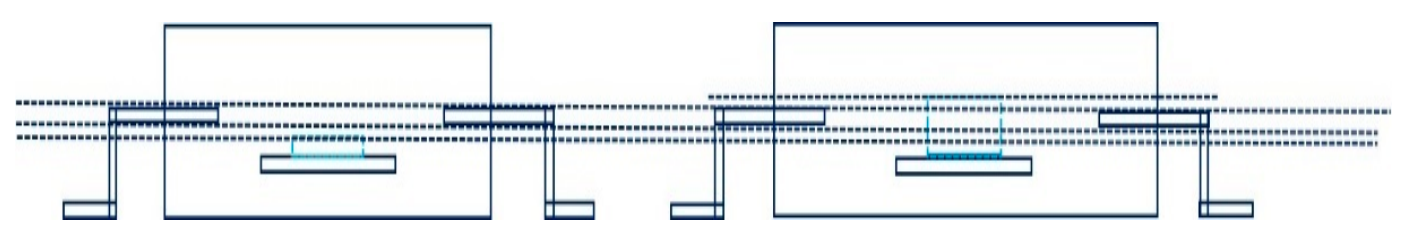

Figure 3. Schematic representation of a non-Manhattan transformation for a pLQFP, where the die (blue rectangle) thickness increases.
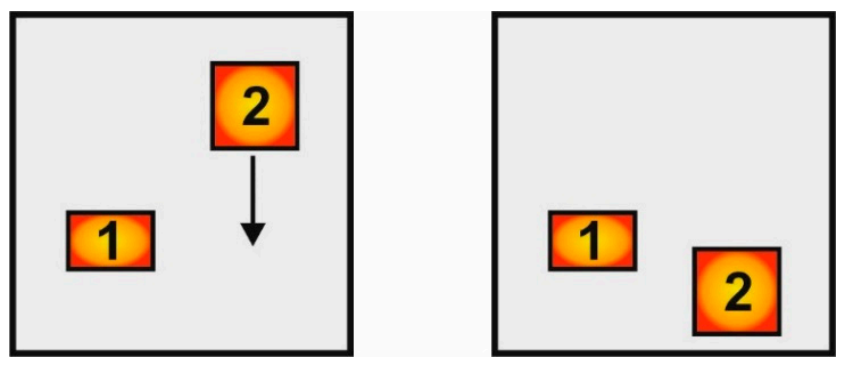

Figure 4. Sketch of a non-Manhattan transformation for a multi-source die.

The functioning principle of TRIC can be described as follows. The DTM of a specimen in a family of packages immersed in a standard environment, automatically extracted by TRIC using the FV method, has the following form:

$$
\mathbf{M}(\mathbf{p}) \frac{d \vartheta}{d t}(t, \mathbf{p})+\mathbf{K}(\mathbf{p}) \vartheta(t, \mathbf{p})=\mathbf{G}(\mathbf{p}) \mathbf{P}(t)
$$

in which $\vartheta(t, \mathbf{p})$ is the $N(\mathbf{p})$ rows vector with the DoF of temperature rise at each time instant $t, \mathbf{M}(\mathbf{p})$ is the $N(\mathbf{p})$-order mass matrix, $\mathbf{K}(\mathbf{p})$ is the $N(\mathbf{p})$-order stiffness matrix, $\mathbf{G}(\mathbf{p})$ is the $N(\mathbf{p}) \times M$ power density rectangular matrix, and $\mathbf{P}(t)$ is the $M$ rows vector of source powers. 
In the first step, a basis for the projection of (2) is determined by Algorithm 1 exploiting Algorithm 2. In the second step, using this projection basis, a fast solution of (2) is provided for a chosen value of $\mathbf{p}$ by Algorithm 3, which again exploits Algorithm 2.

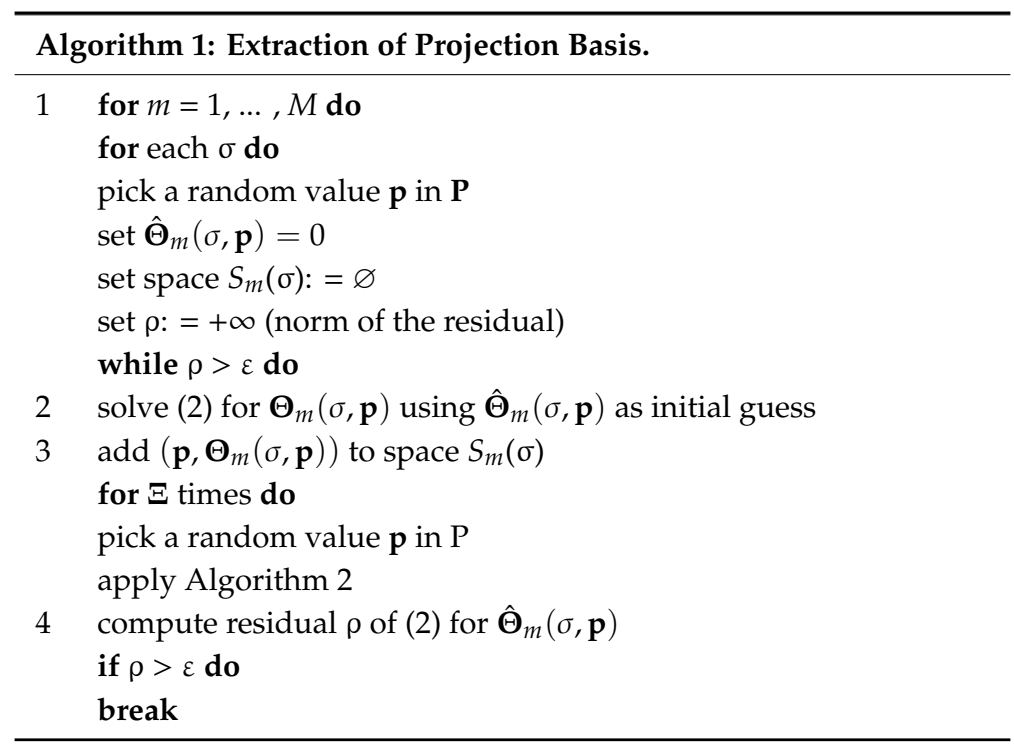

In Algorithm 1, at line 1, a set of complex frequency values, proper for characterizing the thermal behavior of the family of packages, is chosen following [13]. At line 2, the detailed thermal problem in the complex frequency domain

$$
[\sigma \mathbf{M}(\mathbf{p})+\mathbf{K}(\mathbf{p})] \boldsymbol{\Theta}_{m}(\sigma, \mathbf{p})=\mathbf{g}_{m}
$$

in which $\mathbf{g}_{\mathrm{m}}$ is the $m$-th column of $\mathbf{G}$ and is numerically solved by an iterative solver. A multigrid solver is used, and the number of iterations is reduced by assuming as initial guess the $\hat{\boldsymbol{\Theta}}_{m}(\sigma, \mathbf{p})$ estimation. At line 3, the solutions $\boldsymbol{\Theta}_{m}(\sigma, \mathbf{p})$ are added to $S(\sigma)$. At line 4 , the residual $\rho$ is determined substituting $\boldsymbol{\Theta}_{m}(\sigma, \mathbf{p})$ with $\hat{\boldsymbol{\Theta}}_{m}(\sigma, \mathbf{p})$ in (3).

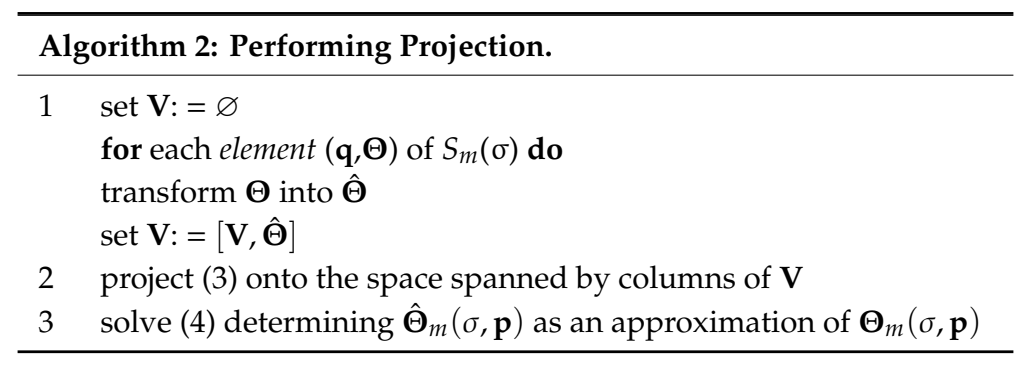

In Algorithm 2, at line 1, the spatial geometry of the DTM for value $\mathbf{p}$ of the parameter vector is expressed as a map of the spatial geometry of the DTM for value $\mathbf{q}$ of the parameters vector. This map is thus applied to vector $\boldsymbol{\Theta}$, getting vector $\hat{\boldsymbol{\Theta}}$. At line 2 , the projected equations are

$$
(\sigma \hat{\mathbf{M}}+\hat{\mathbf{K}}) \hat{\xi}_{m}(\sigma)=\hat{\mathbf{g}}_{m}
$$

where $\hat{\mathbf{M}}$ and $\hat{\mathbf{K}}$ are projected matrices given by $\hat{\mathbf{M}}=\hat{\mathbf{V}}^{T} \mathbf{M}(\mathbf{p}) \hat{\mathbf{V}}$ and $\hat{\mathbf{K}}=\hat{\mathbf{V}}^{T} \mathbf{K}(\mathbf{p}) \hat{\mathbf{V}}$, while $\hat{\xi}_{m}$ is the DoF column vector and $\hat{\mathbf{g}}_{m}=\hat{\mathbf{V}}^{T} \mathbf{g}_{m}$. At line 3, vector $\boldsymbol{\Theta}_{m}(\sigma, \mathbf{p})$ is approximated by

$$
\hat{\boldsymbol{\Theta}}_{m}(\sigma, \mathbf{p})=\hat{\mathbf{V}} \hat{\boldsymbol{\xi}}_{m}(\sigma)
$$


In Algorithm 3, for a chosen value of $\mathbf{p}$, a space $S(\mathbf{p})$ is determined. Projecting (2) onto such a space, the CTM ensues, the response of which to any power profile is computed for approximating $\vartheta(t, \mathbf{p})$.

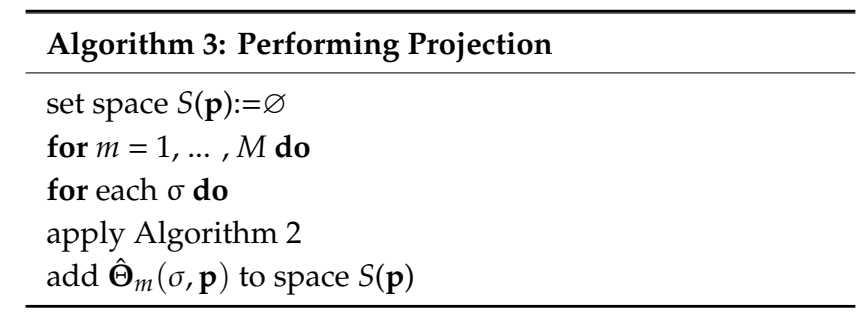

It must be remarked that in commercial numerical codes the complexity for the transient solutions is proportional to $\left(\mathrm{n}_{\mathrm{x}} \times \mathrm{n}_{\mathrm{y}} \times \mathrm{n}_{\mathrm{z}}\right)^{\alpha \times \mathrm{nt}}$, where $\alpha$ is in the range $1 \div 1.5$ (depending on the iterative method adopted); $n_{x}, n_{y}$, and $n_{z}$ are the numbers of grid points along $x, y$, and $z$, and $n_{t}$ is the number of time instants in which the problem has to be solved. Conveniently, the corresponding complexity in TRIC is proportional to $n_{t}$ and independent of $n_{x}, n_{y}$, and $n_{z}$.

\section{Numerical Results}

Except for the multi-source analysis (Section 4.4), the intrinsic symmetry of the packages under test allowed meshing and simulating only a quarter of each structure, thus mitigating the computational burden; the missing portions were virtually restored by applying adiabatic BCs (i.e., zero heat flux) over the planes of symmetry.

\subsection{Full-Plastic LQFP vs. Epad LQFP}

Figure 5 shows the static thermal metrics $\vartheta_{\text {JA }}$ and $\vartheta_{\text {JCtop }}$ corresponding to eLQFPs and pLQFPs for two horizontal package sizes, namely (a) $10 \times 10$ and (b) $14 \times 14 \mathrm{~mm}^{2}$, the epad size being $6 \times 6$ and $7.2 \times 7.2 \mathrm{~mm}^{2}$ for cases (a) and (b), respectively. The metrics are determined by TRIC for various sizes of the square die, i.e., $2 \times 2,3 \times 3,4 \times 4,5 \times 5,6 \times 6 \mathrm{~mm}^{2}$, the latter only for case (b). It can be inferred that the presence of the pad, which eases the heat removal, yields a sizable beneficial impact on $\vartheta_{\mathrm{JA}}$, whereas the closer proximity of the die to the top of the package in pLQFPs prevails over the cooling action of the epad in terms of $\vartheta_{\text {JCtop. }}$.
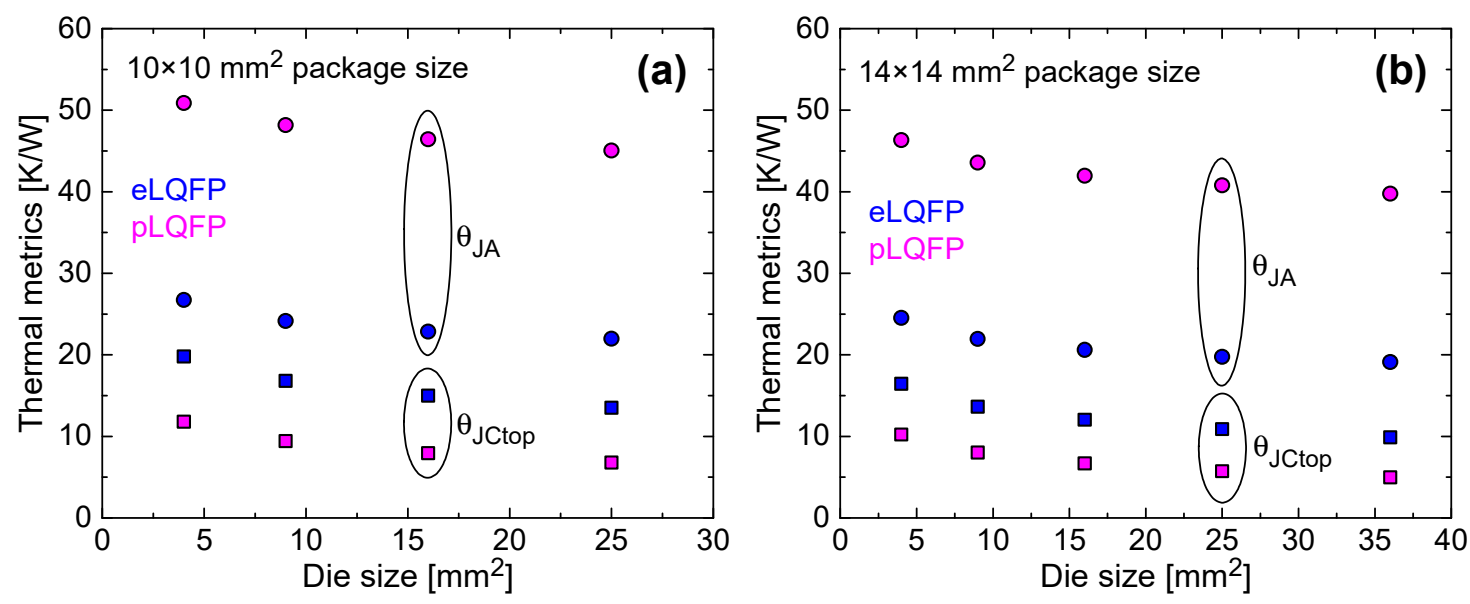

Figure 5. Thermal metrics $\vartheta_{\text {JA }}$ (circles) and $\vartheta_{\text {JCtop }}$ (squares) against die size: comparison between eLQFP (blue) and pLQFP (magenta) for (a) $10 \times 10$ and (b) $14 \times 14 \mathrm{~mm}^{2}$ package size with (a) $6 \times 6 \mathrm{~mm}^{2}$ and (b) $7.2 \times 7.2 \mathrm{~mm}^{2}$ die sizes. 


\subsection{Thermal Impedances}

One of the main features of TRIC not available in TRAC is the possibility to determine the transient thermal responses of a specimen of a package family for any profile of the power sources. In particular, the thermal impedances-often used to characterize the dynamic thermal behavior of components-can be evaluated as the thermal responses (temperature rises normalized to power) to a power step applied at $t=0$ to a die modeled with only one HS. Such a capability is witnessed through the analysis of a large set of cases.

Figure 6 shows the junction-to-ambient thermal impedance $Z_{T H J A}=\vartheta_{J A}(t)$ of a $10 \times 10 \mathrm{~mm}^{2}$ eTQFP equipped with a $6 \times 6 \mathrm{~mm}^{2}$ epad for four different die sizes. The simulations allow quantifying the favorable influence of a large die, which benefits from a lower power density. Static conditions are reached at about $400 \mathrm{~s}$, regardless of die size. Figure 7 illustrates the $Z_{\text {THJA }}$ of a $10 \times 10 \mathrm{~mm}^{2}$ pLQFP with a $4 \times 4 \mathrm{~mm}^{2}$ die for three different pad sizes. All curves coincide for short times $(<0.1 \mathrm{~s})$, where the heat emerging from the die has not hit the pad yet. For this case, (i) a complex evolution with an inflection takes place due to the involved package geometry: the heat propagates through the pad, the mold, and then reaches the leads, which are in direct contact with the board, and (ii) the impedances flatten at $1200 \mathrm{~s}$, as induced by the absence of the cooling epad action. Figure 8 reports the $Z_{\text {THJA }}$ of $9 \times 9 \mathrm{~mm}^{2}$ single-row eQFN packages for three combinations of epad and die sizes. Here the positive influence of a bigger epad $\left(7 \times 7 \mathrm{~mm}^{2}\right.$ instead of $\left.5.7 \times 5.7 \mathrm{~mm}^{2}\right)$ for the same die size is evident; again, the impedances overlap for short times. Similar to the study conducted for the eTQFP family, also for eQFN packages the thermal impedance is reduced and delayed for bigger dies. Figure 9 confirms the cooling impact for medium/long times of a larger package body and/or a larger epad for eQFN-mr packages with two rows of pins. Lastly, the dynamic thermal behavior of a $10.3 \times 7.5 \mathrm{~mm}^{2}$ PowerSSO-36 with a $4.09 \times 3.17 \mathrm{~mm}^{2}$ die is determined for four different epad sizes in Figure 10 .

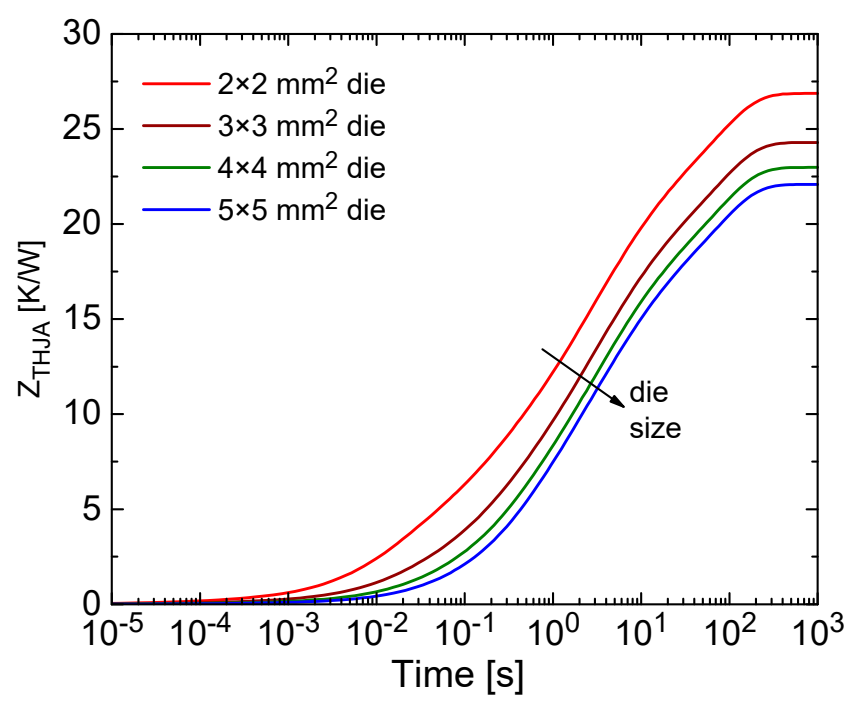

Figure 6. Thermal impedance $Z_{\text {THJA }}$ vs. time of a $10 \times 10 \mathrm{~mm}^{2}$ eTQFP with a $6 \times 6 \mathrm{~mm}^{2}$ epad for four different die sizes. 


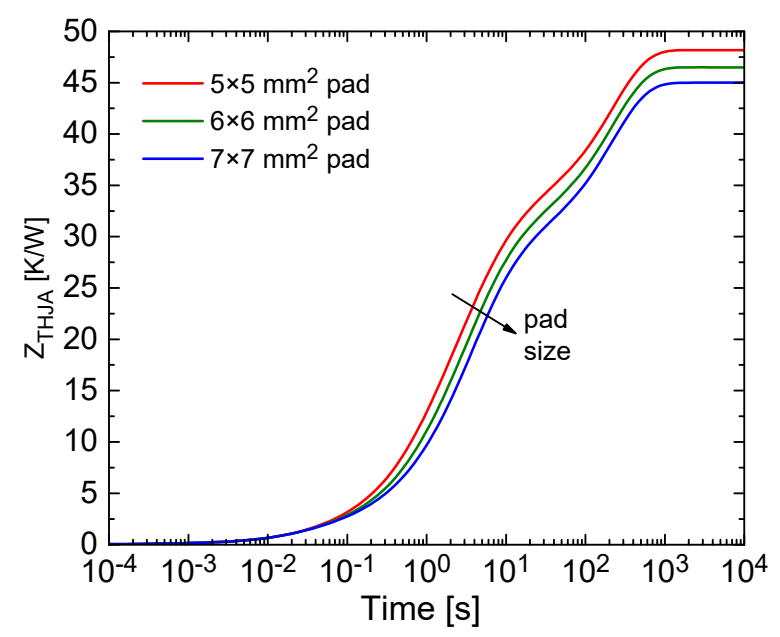

Figure 7. Thermal impedance $Z_{\text {THJA }}$ against time of a $10 \times 10 \mathrm{~mm}^{2}$ pLQFP with a $4 \times 4 \mathrm{~mm}^{2}$ die for three different pad sizes.

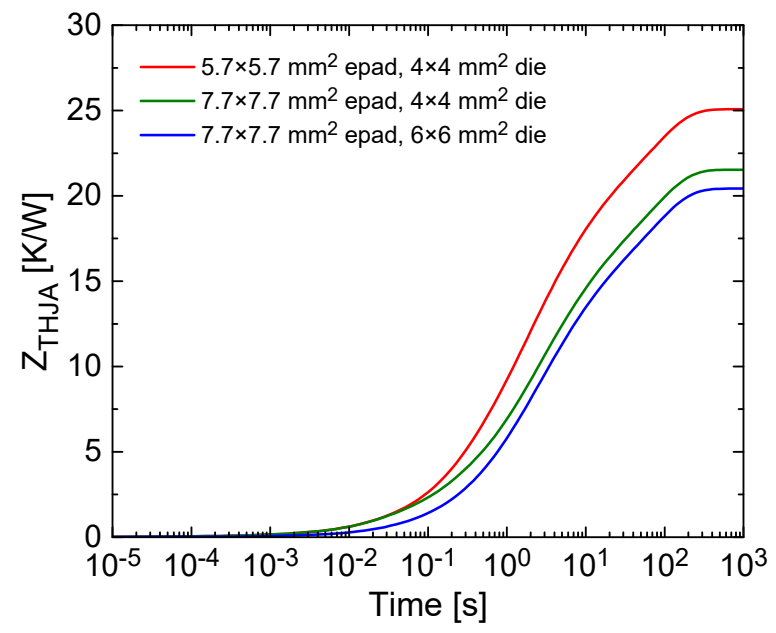

Figure 8. Thermal impedance $Z_{\text {THJA }}$ vs. time of a $9 \times 9 \mathrm{~mm}^{2}$ single-row eQFN package for three combinations of epad and die sizes.

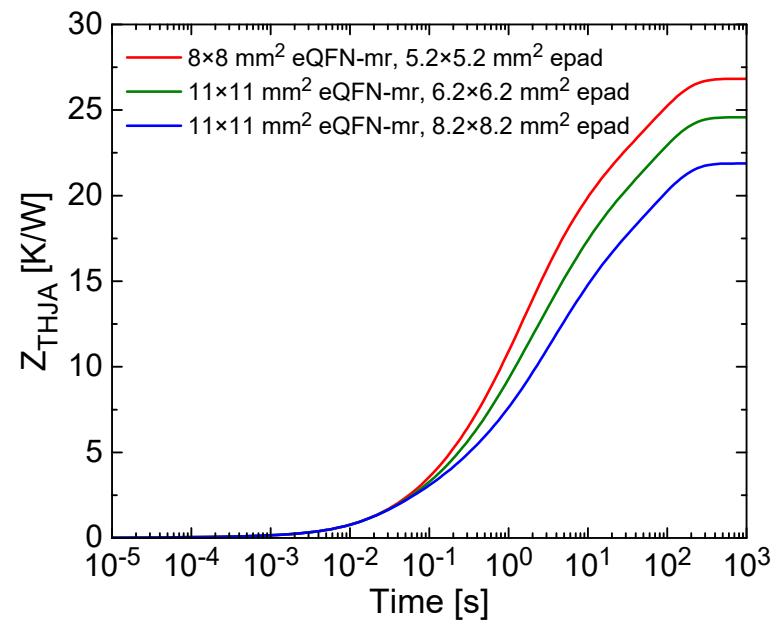

Figure 9. Thermal impedance $\mathrm{Z}_{\mathrm{THJ}}$ vs. time of various dual-row eQFN-mr packages sharing a die size of $3 \times 3 \mathrm{~mm}^{2}$. 


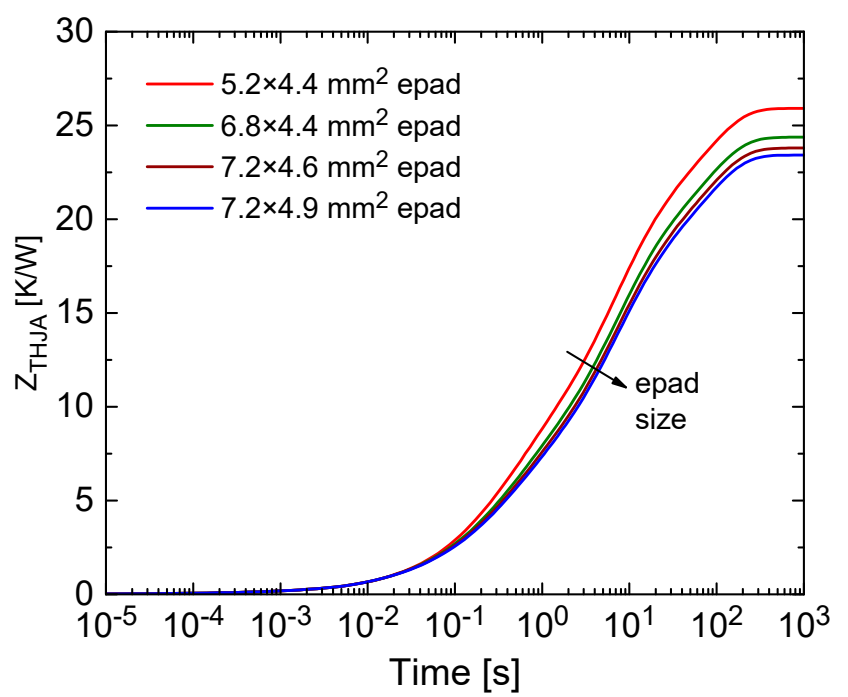

Figure 10. Thermal impedance $\mathrm{Z}_{\mathrm{THJA}}$ of a $10.3 \times 7.5 \mathrm{~mm}^{2}$ PowerSSO-36 L with a $4.09 \times 3.17 \mathrm{~mm}^{2}$ die for four different epad sizes.

\subsection{Comparison with FloTHERM}

First and foremost, it must be underlined that the user-friendly graphical interface of TRIC allows avoiding the long, painstaking, and prone-to-error geometry/mesh construction process often required by conventional numerical tools, thus markedly lowering the pre-processing effort and time. As far as the accuracy and efficiency of TRIC are concerned, they were estimated by comparison with the widely used commercial software FloTHERM. The tools share the same geometry simplifications and BCs. The favorable matching between the thermal metrics determined by TRAC and the FV software was already shown in [11] for eLQFPs, eTQFPs, and eQFN packages; the slight discrepancy (typically $<2 \%$, the maximum value being around 3\%) was mainly attributed to the different mesh styles of the simulators. Evidence of the good agreement is also provided in Figure 11, which shows the (static) maps of temperature rise over ambient $\left(\mathrm{T}_{\mathrm{amb}}=20^{\circ} \mathrm{C}\right)$ determined for three eTQFPs with dies dissipating $1 \mathrm{~W}$ in the $\vartheta_{\mathrm{JA}}$-related ambient; in particular, (a) corresponds to a $14 \times 14 \mathrm{~mm}^{2}$ package size with a $9 \times 9 \mathrm{~mm}^{2}$ epad and a $3 \times 3 \mathrm{~mm}^{2}$ die; (b) to a $10 \times 10 \mathrm{~mm}^{2}$ package with a $6 \times 6 \mathrm{~mm}^{2}$ epad and a $4 \times 4 \mathrm{~mm}^{2}$ die; (c) to a $10 \times 10 \mathrm{~mm}^{2}$ package with a $6 \times 6 \mathrm{~mm}^{2}$ epad and a $2 \times 2 \mathrm{~mm}^{2}$ die.

The accuracy ensured by TRIC under transient conditions can be inferred from Figure 12, which shows the favorable matching with data computed by FloTHERM for three packages belonging to the eTQFP family. Again, the discrepancy is below $3 \%$ within the whole time range. It is worth noting that the CPU time required to obtain an impedance by TRIC for a typical number of $2 \times 10^{6}$ grid points is about 1 min on a workstation with an Intel Xeon E5-2630 v4@ $2.2 \mathrm{GHz}$ equipped with a 64 GB RAM, whereas more than 15-20 minutes are needed when using FloTHERM. This notable gain in terms of efficiency is expected to take place with respect to most popular simulators based on numerical methods. 

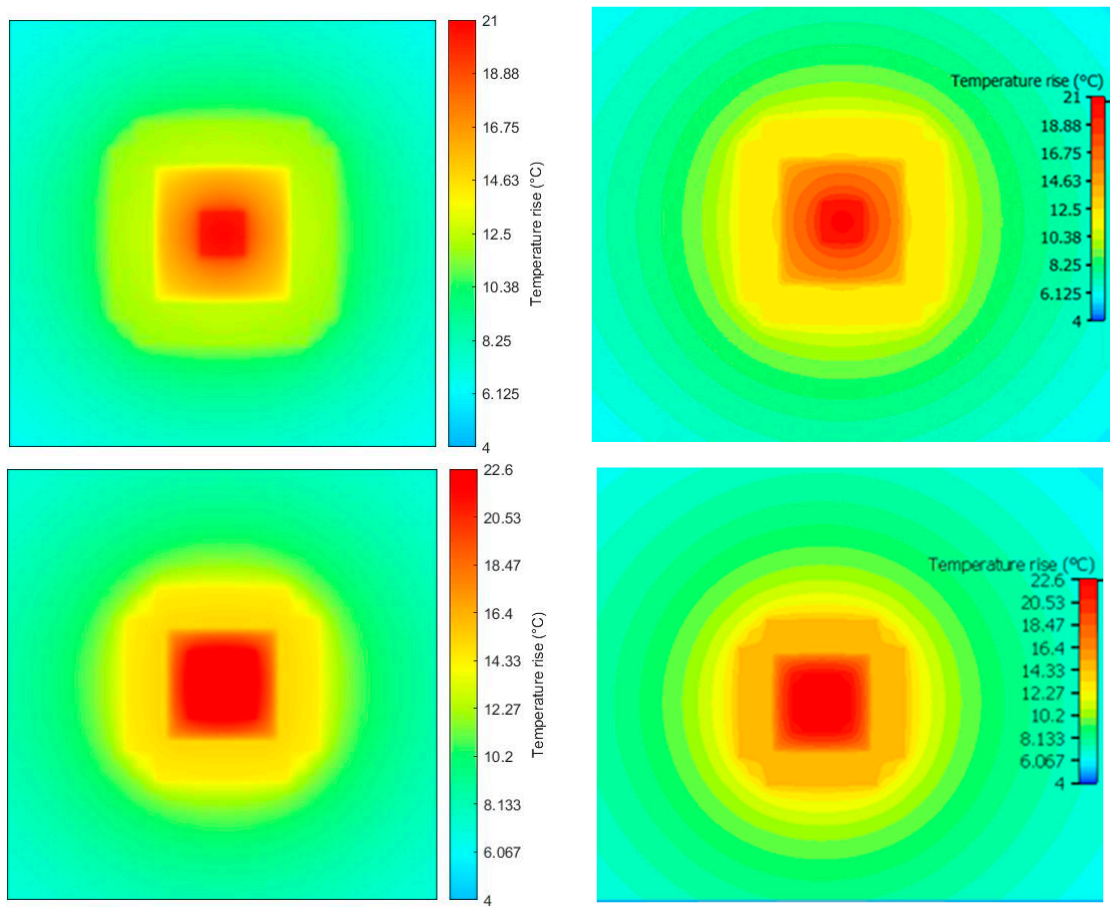

(a)

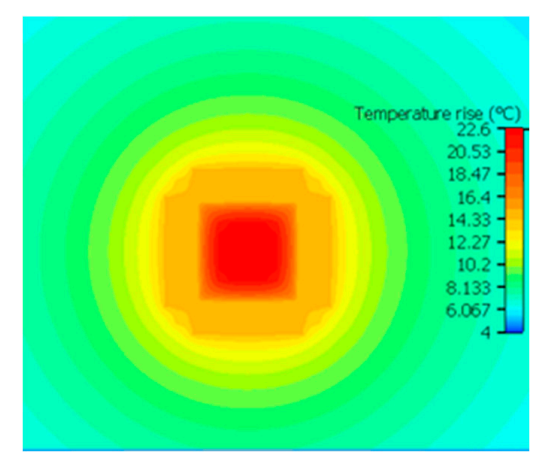

(b)
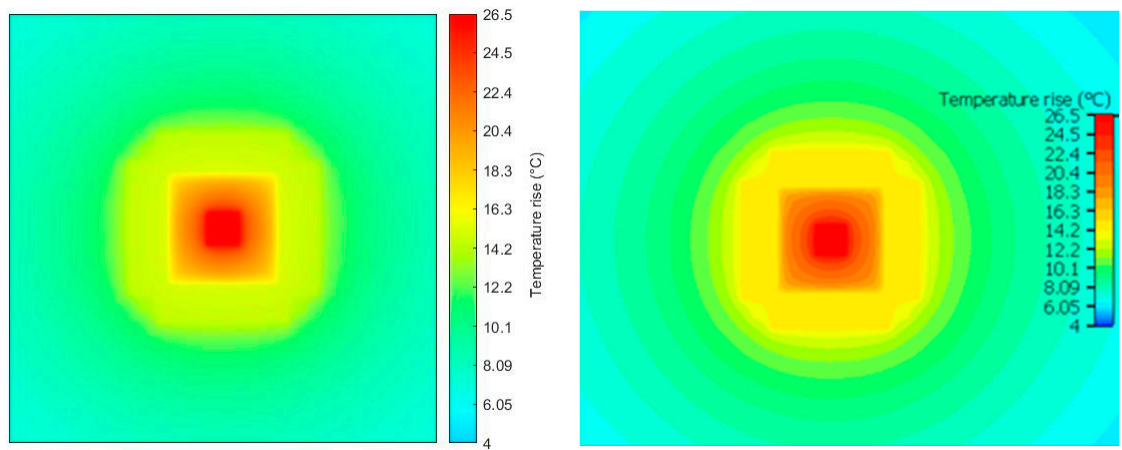

(c)

Figure 11. Temperature rise maps for three eTQFPs dissipating $1 \mathrm{~W}$, as calculated by TRIC (left) and FloTHERM (right): (a) $14 \times 14 \mathrm{~mm}^{2}$ package size with a $9 \times 9 \mathrm{~mm}^{2}$ epad and a $3 \times 3 \mathrm{~mm}^{2}$ die; (b) $10 \times 10 \mathrm{~mm}^{2}$ package with a $6 \times 6 \mathrm{~mm}^{2}$ epad and a $4 \times 4 \mathrm{~mm}^{2}$ die; (c) $10 \times 10 \mathrm{~mm}^{2}$ package with a $6 \times 6 \mathrm{~mm}^{2}$ epad and a $2 \times 2 \mathrm{~mm}^{2}$ die.
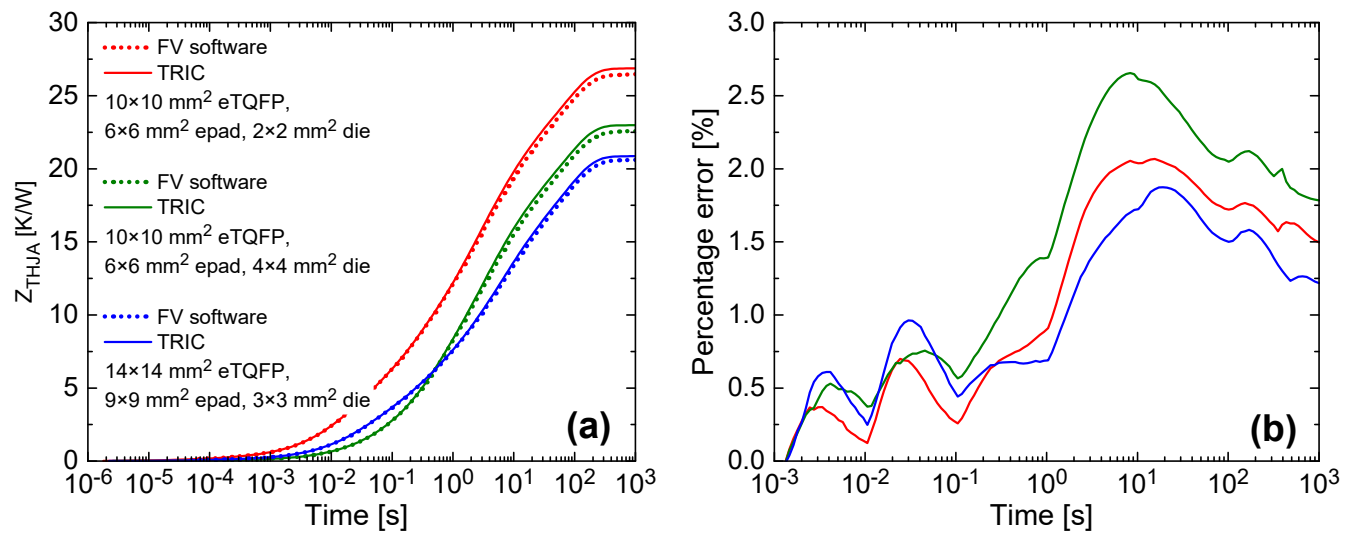

Figure 12. (a) Comparison between TRIC and FloTHERM for various eTQFP cases; (b) corresponding percentage error vs. time. 


\subsection{Multi-Source Analysis}

Unlike TRAC, TRIC allows also simulating realistic packages that integrate multiple HSs, as evidenced through the following illustrative examples.

First, a $9 \times 9 \mathrm{~mm}^{2}$ eQFN package is considered, with a $5 \times 5 \mathrm{~mm}^{2}$ epad and a $4 \times 4 \mathrm{~mm}^{2}$ die, the latter including four $1 \times 1 \mathrm{~mm}^{2}$ active areas (i.e., HSs), each dissipating $0.5 \mathrm{~W}$. As sketched in Figure 13, four positions of the HSs are chosen to describe practical layouts. The static temperature rise over ambient was monitored in five critical points, namely, at the die center $\left(\Delta \mathrm{T}_{\text {center }}\right)$ and at the centers of the HSs $\left(\Delta \mathrm{T}_{1}, \Delta \mathrm{T}_{2}, \Delta \mathrm{T}_{3}, \Delta \mathrm{T}_{4}\right)$. Results corresponding to the four layouts are reported in Table 1, along with the maximum temperature rise over the whole die $\left(\Delta \mathrm{T}_{\max }\right)$. Again, a fairly good agreement with the temperature maps determined by FloTHERM (not shown here) was obtained, the discrepancy between the maxima being $<3 \%$. The data plainly illustrate how the temperature field over the die modifies depending on the specific layout. The main findings are (i) all the HSs share the same temperature in cases \#1 and \#3 for symmetry reasons; (ii) as expected, in layout \#1 $\Delta \mathrm{T}_{\text {center }}$ $=\Delta \mathrm{T}_{\max }$ due to the concurrent influence of all the HSs, while (iii) $\Delta \mathrm{T}_{\max }$ is reached near the die side in layout \#2. This simple analysis shows that TRIC can be effectively exploited to identify the most thermally efficient layout. In addition, the information gained on the temperature field over the die is also important to properly place temperature sensors.

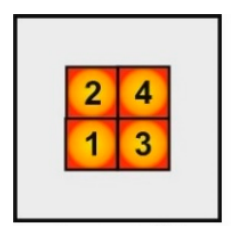

layout \#1

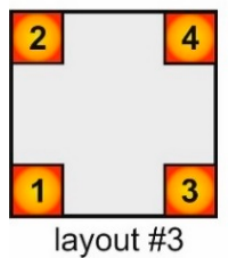

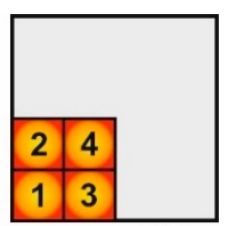

layout \#2

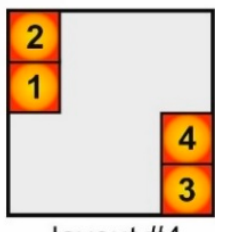

layout \#4

Figure 13. Representation of four layouts corresponding to a four-source die. The temperature-sensing points are located at the center of the die and at the centers of the HSs.

Table 1. Temperature rises over ambient (K) computed by TRIC.

\begin{tabular}{lcccccc}
\hline & $\boldsymbol{\Delta} \mathbf{T}_{\mathbf{1}}$ & $\boldsymbol{\Delta} \mathbf{T}_{\mathbf{2}}$ & $\boldsymbol{\Delta} \mathbf{T}_{\mathbf{3}}$ & $\boldsymbol{\Delta} \mathbf{T}_{\mathbf{4}}$ & $\boldsymbol{\Delta} \mathbf{T}_{\text {center }}$ & $\Delta \mathbf{T}_{\max }$ \\
\hline Layout \#1 & 56.31 & 56.31 & 56.31 & 56.31 & 57.06 & 57.06 \\
\hline Layout \#2 & 59.56 & 58.20 & 58.20 & 57.05 & 54.13 & 59.58 \\
\hline Layout \#3 & 54.89 & 54.89 & 54.89 & 54.89 & 52.25 & 55.15 \\
\hline Layout \#4 & 55.77 & 56.12 & 56.12 & 55.77 & 52.70 & 56.35 \\
\hline
\end{tabular}

\subsection{ABS Source Profile}

The TRIC capability to cover complex die layouts and transient power profiles is demonstrated by simulating the die temperature dictated by a realistic ABS power profile. The examined package is a $14 \times 14 \mathrm{~mm}^{2}$ eTQFP with an $8 \times 8 \mathrm{~mm}^{2}$ epad and a $30 \mathrm{~mm}^{2}$ die, the circuitry over which presents eight active areas (HSs), as depicted in Figure 14a. The geometry of the system and the external BCs were adapted to this specific application. Figure 14b illustrates the TRIC interface with the probes (placed at the centers of the HSs) where the temperatures are taken. Figure $14 \mathrm{c}$ shows the evolution of the temperature rises over ambient from 0 to $20 \mathrm{~s}$. Figure $14 \mathrm{~d}$ reports the temperature rise field at the most thermally critical time instant, i.e., $2.5 \mathrm{~s}$. 


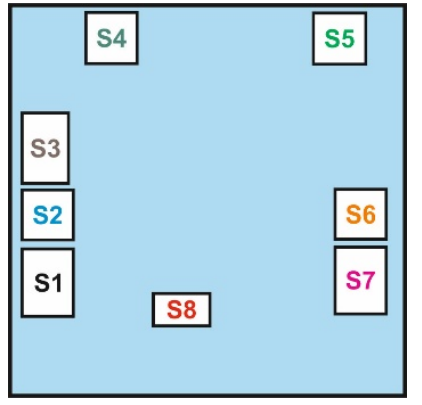

(a)

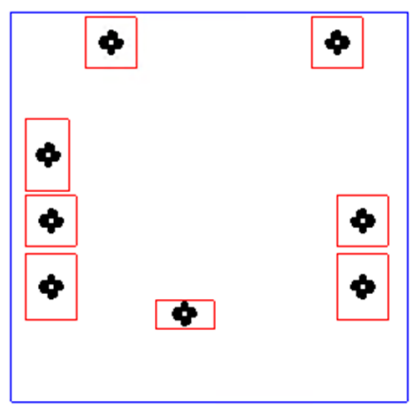

(b)
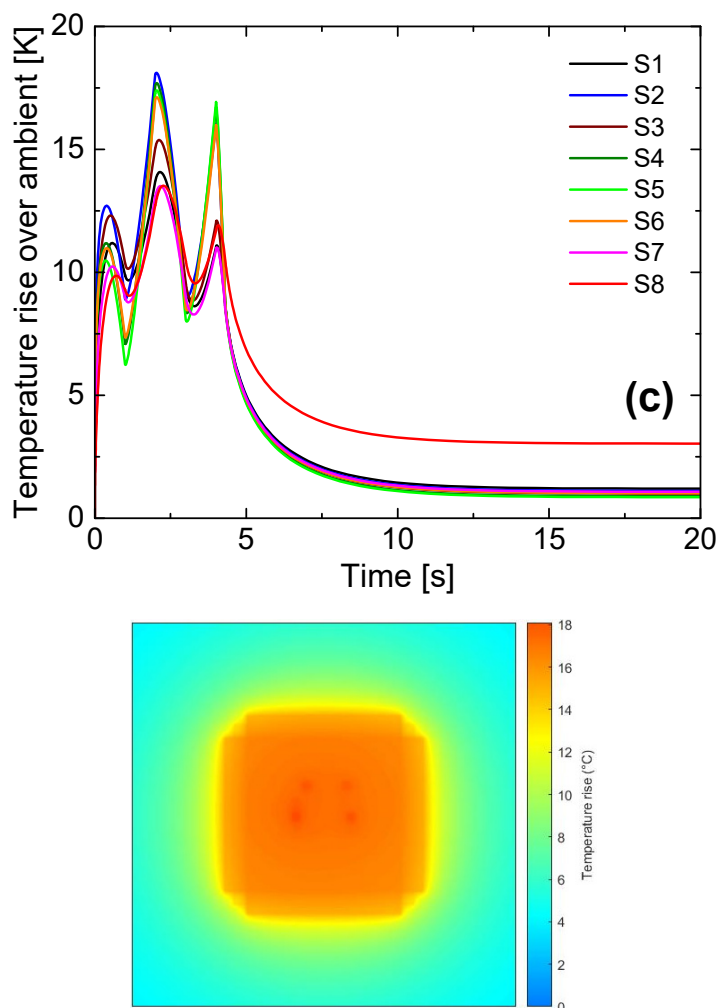

(d)

Figure 14. (a) Schematic top-view of the die floorplan; (b) corresponding TRIC interface; (c) evolution of the temperature rises vs. time; $(\mathbf{d})$ temperature rise map at the time instant $t=2.5 \mathrm{~s}$.

\section{Conclusions}

In this paper, a tool denoted as Thermal Resistance and Impedance Calculator (TRIC) has been presented. TRIC allows the automatic extraction of thermal metrics of package families of electronic components under both static and transient conditions. It exploits a solution algorithm based on a novel projection-based approach, which allows dealing with non-Manhattan geometry and mesh variations in the parametric detailed thermal model (pDTM) of a package family. The pDTMs of many relevant package families have been included, and dies with multiple active areas can be handled. An extensive simulation campaign, focused on cases of practical interest, has been performed. A comparison between TRIC and the FV program FloTHERM has been carried out, with the aim of validating the accuracy and assessing the efficiency of the proposed tool; the main findings can be summarized as follows: (i) the discrepancy in terms of thermal metrics calculated by the simulators amounts at most to $3 \%$ and is mainly ascribable to the different mesh styles; (ii) thanks to its advanced solution algorithm, TRIC allows obtaining a reduction in CPU time by a factor of 15-20 with respect to FloTHERM when simulating a transient thermal response. Owing to the above reasons, TRIC can 
be considered particularly helpful for industry specialists who are involved in designing packaged devices and have to cope with thermal flow problems.

Author Contributions: Methodology, L.C.; Software, L.C., F.D.V., D.G., A.M., and C.M.V.; Validation, F.D.V. and L.C.; Writing-Original Draft Preparation, V.d.; Writing-Review \& Editing, V.d.; Supervision, L.C. and C.M.V. All authors have read and agreed to the published version of the manuscript.

Funding: This research received no external funding.

Conflicts of Interest: The authors declare no conflict of interest.

\section{Nomenclature}

TRIC

TRAC

JEDEC

thermal resistance $(\mathrm{K} / \mathrm{W})$

thermal impedance $(\mathrm{K} / \mathrm{W})$

$\vartheta_{\text {JA }}(\mathrm{K} / \mathrm{W})$

$\Psi_{\mathrm{JB}}(\mathrm{K} / \mathrm{W})$

$\Psi_{\text {JCtop }}(\mathrm{K} / \mathrm{W})$

$\vartheta_{\mathrm{JB}}(\mathrm{K} / \mathrm{W})$

$\vartheta_{\text {JCtop }}(\mathrm{K} / \mathrm{W})$

$\vartheta_{\text {JCbottom }}(\mathrm{K} / \mathrm{W})$

$\mathrm{Z}_{\text {THJA }}(\mathrm{K} / \mathrm{W})$

$\mathrm{BC}$

BCI

MOR

CTM

DTM

pDTM

FV

DoF

HS

CPU

epad

QFP

eLQFP

eTQFP

pLQFP

QFN

eQFN

eQFN-mr

PowerSSO

ABS
Thermal Resistance and Impedance Calculator

Thermal Resistance Advanced Calculator

Joint Electron Device Engineering Council

temperature increase over a reference temperature taken over a

point (or a region) of interest of the component under test, and

normalized to the dissipated power; it is a property depending

upon geometry and material parameters, and can be reviewed as

an indicator of the heat dissipation inaptitude of the component

thermal resistance vs. time resulting from the application of a

constant power step

junction-to-ambient thermal resistance

thermal characterization parameter to report the difference

between junction temperature and the temperature of the board

measured at the top surface of the board

thermal characterization parameter to report the difference

between junction temperature and the temperature at the top

center of the outside surface of the component package

junction-to-board thermal resistance

junction-to-case top thermal resistance

junction-to-case bottom thermal resistance

junction-to-ambient thermal impedance

boundary condition

boundary condition independent

model-order reduction

compact thermal model

detailed thermal model

parametric DTM

finite volume

degree of freedom

heat source

central processing unit

exposed pad

quad flat package

exposed-pad low-profile (thick) QFP

exposed-pad thin QFP

full-plastic low-profile (thick) QFP

quad flat no-leads package

exposed-pad QFN

multi-row eQFN

package belonging to the Small Outline family

antilock braking system 


\section{References}

1. Smy, T.; Walkey, D.; Dew, S.K. A 3D thermal simulation tool for integrated devices-Atar. IEEE Trans. Comput. Aided Des. Integr. Circuits Syst. 2001, 20, 105-115. [CrossRef]

2. Huang, W.T.; Ghosh, S.; Sankaranarayanan, K.; Skadron, K.; Stan, M.R. Hotspot: Thermal modeling for CMOS VLSI systems. IEEE Trans. Large Scale Integr. VLSI Syst. 2006, 14, 501-513. [CrossRef]

3. Ziabari, A.; Park, J.H.; Ardestani, E.K.; Renau, J.; Kang, S.M.; Shakouri, A. Power blurring: Fast static and transient thermal analysis method for packaged integrated circuits and power devices. IEEE Trans. Large Scale Integr. VLSI Syst. 2014, 22, 2366-2379. [CrossRef]

4. Bar-Cohen, A.; Elperin, T.; Eliasi, R. $\vartheta_{\text {JC }}$ characterization of chip packages - justification, limitations, and future. IEEE Trans. Compon. Hybrids Manuf. Technol. 1989, 12, 724-731. [CrossRef]

5. Lasance, C.J.M.; Vinke, H.; Rosten, H. Thermal characterization of electronic devices with boundary condition independent compact models. IEEE Trans. Compon. Packag. Manuf. Technol. Part A 1995, 18, $723-731$. [CrossRef]

6. Pape, H.; Schweitzer, D.; Janssen, J.H.J.; Morelli, A.; Villa, C.M. Thermal transient modeling and experimental validation in the European project PROFIT. IEEE Trans. Compon. Packag. Technol. 2004, 27, 530-538. [CrossRef]

7. Sabry, M.N. Flexible profile compact thermal models for practical geometries. J. Electr. Packag. 2007, 129, 256-259. [CrossRef]

8. Lasance, C.J.M. Ten years of boundary-condition-independent compact thermal modeling of electronic parts: A review. Heat Transf. Eng. 2008, 29, 149-169. [CrossRef]

9. JESD51-12. Guidelines for Reporting and Using Electronic Package Thermal Information; JEDEC: Arlington, VA, USA, May 2005.

10. Codecasa, L.; Race, S.; d'Alessandro, V.; Gualandris, D.; Morelli, A.; Villa, C.M. Thermal resistance advanced calculator (TRAC). In Proceedings of the International Workshop on THERMal INvestigation of ICs and Systems (THERMINIC), Stockholm, Sweden, 26-28 September 2018.

11. Codecasa, L.; Race, S.; d'Alessandro, V.; Gualandris, D.; Morelli, A.; Villa, C.M. TRAC: A thermal resistance advanced calculator for electronic packages. Energies 2019, 12, 1050. [CrossRef]

12. Codecasa, L.; D'Amore, D.; Maffezzoni, P. Modeling the thermal response of semiconductor devices through equivalent electrical networks. IEEE Trans. Circuits Syst. I Fundam. Theory Appl. 2002, 49, 1187-1197. [CrossRef]

13. Codecasa, L.; D'Amore, D.; Maffezzoni, P. Compact modeling of electrical devices for electrothermal analysis. IEEE Trans. Circuits Syst. I Fundam. Theory Appl. 2003, 50, 465-476. [CrossRef]

14. Codecasa, L.; D'Amore, D.; Maffezzoni, P. Multipoint moment matching reduction from port responses of dynamic thermal networks. IEEE Trans. Compon. Packag. Technol. 2005, 28, 605-614. [CrossRef]

15. Codecasa, L.; d'Alessandro, V.; Magnani, A.; Rinaldi, N.; Zampardi, P.J. FAst novel thermal analysis simulation tool for integrated circuits (FANTASTIC). In Proceedings of the International Workshop on THERMal INvestigation of ICs and systems (THERMINIC), London, UK, 24-26 September 2014.

16. Codecasa, L.; d'Alessandro, V.; Magnani, A.; Rinaldi, N. Parametric compact thermal models by moment matching for variable geometry. In Proceedings of the International Workshop on THERMal INvestigation of ICs and systems (THERMINIC), London, UK, 24-26 September 2014.

17. Magnani, A.; d'Alessandro, V.; Codecasa, L.; Zampardi, P.J.; Moser, B.; Rinaldi, N. Analysis of the influence of layout and technology parameters on the thermal impedance of GaAs HBT/BiFET using a highly-efficient tool. In Proceedings of the IEEE Compound Semiconductor Integrated Circuit Symposium (CSICS), La Jolla, CA, USA, 19-22 October 2014.

18. Codecasa, L.; d'Alessandro, V.; Magnani, A.; Rinaldi, N. Matrix reduction tool for creating boundary condition independent dynamic compact thermal models. In Proceedings of the International Workshop on THERMal INvestigation of ICs and Systems (THERMINIC), Paris, France, 30 September-2 October 2015.

19. Janssen, J.H.J.; Codecasa, L. Why matrix reduction is better than objective function based optimization in compact thermal model creation. In Proceedings of the International Workshop on THERMal Investigation of ICs and Systems (THERMINIC), Paris, France, 30 September-2 October 2015.

20. Codecasa, L.; d'Alessandro, V.; Magnani, A.; Rinaldi, N. Structure preserving approach to parametric dynamic compact thermal models of nonlinear heat conduction. In Proceedings of the International Workshop on THERMal INvestigation of ICs and Systems (THERMINIC), Paris, France, 30 September-2 October 2015. 
21. Codecasa, L.; d'Alessandro, V.; Magnani, A.; Irace, A. Circuit-based electrothermal simulation of power devices by an ultrafast nonlinear MOS approach. IEEE Trans. Power Electron. 2016, 31, 5906-5916. [CrossRef]

22. Rogié, B.; Codecasa, L.; Monier-Vinard, E.; Bissuel, V.; Laraqi, N.; Daniel, O.; D'Amore, D.; Magnani, A.; d'Alessandro, V.; Rinaldi, N. Delphi-like dynamical compact thermal models using model order reduction. In Proceedings of the International Workshop on THERMal INvestigation of ICs and Systems (THERMINIC), Amsterdam, The Netherlands, 27-29 September 2017. (best paper award).

23. Codecasa, L.; d'Alessandro, V.; Magnani, A.; Rinaldi, N. Novel approach for the extraction of nonlinear compact thermal models. In Proceedings of the International Workshop on THERMal INvestigation of ICs and systems (THERMINIC), Amsterdam, The Netherlands, 27-29 September 2017.

24. Codecasa, L.; De Viti, F.; Race, S.; d'Alessandro, V.; Gualandris, D.; Morelli, A.; Villa, C.M. Thermal Resistance and Impedance Calculator (TRIC). In Proceedings of the International Workshop on THERMal INvestigation of ICs and Systems (THERMINIC), Lecco, Italy, 25-27 September 2019.

25. FloTHERM@v12.2. User's Guide; Mentor Graphics: Wilsonville, OR, USA, 2018.

(C) 2020 by the authors. Licensee MDPI, Basel, Switzerland. This article is an open access article distributed under the terms and conditions of the Creative Commons Attribution (CC BY) license (http://creativecommons.org/licenses/by/4.0/). 